\title{
An Angel-Daemon Approach to Asses the Uncertainty in the Power of a Collectivity to Act *
}

\author{
G. Fragnito, J. Gabarro, M. Serna \\ ALBCOM. CS Dept. Universitat Politècnica de Catalunya, Barcelona. \\ giuliafragnito@yahoo.it, \{gabarro,mjserna\}ecs.upc.edu
}

\begin{abstract}
We propose the use of the angel-daemon framework to assess the Coleman's power of a collectivity to act under uncertainty in weighted voting games. First, we introduce a perturbation strength model describing the potential changes in the weights of a weighted game. Based on $\mathcal{S}$, an uncertainty profile $\mathcal{U}$, fixes the spread of the weights' change. In the angel-daemon framework uncertainty profiles describe the potential changes in the weights of a weighted game and fixes the spread of the weights' change. For each uncertainty profile a strategic $\mathfrak{a} / \mathfrak{d}$ game can be considered. The game has two selfish players, the angel $\mathfrak{a}$ and the daemon $\mathfrak{d}, \mathfrak{a}$ selects their action as to minimize the effect on the measure under consideration while $\mathfrak{d}$ acts oppositely. Players $\mathfrak{a}$ and $\mathfrak{d}$ give a balance between the best and the the best and the worst. The $\mathfrak{a} / \mathfrak{d}$ games associated to the Coleman's power are constant sum games and therefore the expected utilities of all the Nash equilibria in the $\mathfrak{a} / \mathfrak{d}$ associated to an uncertainty profile are the same. In this way we can asses the Coleman's power under uncertainty. Besides introducing the framework for this particular setting and analyzing some computational complexity considerations we provide several examples based in the evolution of the voting rules of the EU Council of Ministers.
\end{abstract}

Keywords. Weighted voting games, Coleman's Power of a Collectivity to act, Uncertainty profiles, Strategic games, EU Council of Ministers.

\section{Introduction}

The distinction between risk and uncertainty has become increasingly important since [?] discussed it as we have imperfect knowledge of future events in our ever-changing world. Informally, risk can be measured by probabilities. In contrast, uncertainty refers to something where we cannot even gather the information required to figure out probabilities. However, in practice both are measured by historical standard deviation of the variable of interest [?,?]. This paper proposes an alternative to disentangle these seemingly indistinguishable concepts applying ideas from game theory and computer science.

\footnotetext{
* G. Fragnito is partilly supported by a studentship from La Sapienza. J. Gabarro and M. Serna are partially supported by funds from the Spanish Ministry for Economy and Competitiviness (MIMECO) and the European Union (FEDER funds) under grant COMMAS (ref. TIN201346181-C2-1-R), and also by 2014 SGR 1034 (ALBCOM).
} 
The study of web applications is a field where uncertainty becomes unavoidable. The angel-daemon framework [?] provides a way to obtain numerical estimates of uncertainty in the execution of a Web service. In such a setting, the uncertainty is captured by an uncertainty profile describing a stressed environment for the execution of the Web application. Uncertainty profiles provide a description of the perceived uncertain behaviour with respect to possible failing services or execution delays. That is, some sites can potentially misbehave but we are uncertain about the specific sites that will do so. The model attempts to balance positive and negative aspects. Considering only positive aspects (minimizing damage) is usually too optimistic. In the opposite side, being pessimistic (maximizing damage) is also not realistic. Reality often evolves in between optimism and pessimism. To model this situation, the framework considers two agents : the angel $(\mathfrak{a})$, dealing with the optimistic side; and the daemon $(\mathfrak{d})$, dealing with the pessimistic side. These agents act strategically in an associated angel-daemon zero-sum game. In this context, uncertain situations are identified with the Nash equilibria of the angel-daemon game and they are assessed by the value of the game. It is important to emphasize that the results in [?] are useful to analyse uncertain stable (or timeless) environments. Thus, the framework analyses uncertainty in the short-term and it is not useful for a long-term analysis.

In this paper, we extend the aangel-daemon $(\mathfrak{a} / \mathfrak{d})$ framework to asses uncertainty in a collective measure of power the Coleman's power index [2]. The study of the power of the players inside a weighted voting games [8] is a well developed topic started by Lloyd Shapley in 1953 [7]. Also, the study of the uncertainty in such a weighted voting systems has been considered under a theoretical [3] or practical [6] aspects. Less studied is the study of the power of game as a whole without emphasizing the role of the different players but taking the collectivity as a whole. In 1971 James Coleman [2] introduced the formal definition of the power of a collectivity to act denoted as Act. Practical applications appears in [2]. More recently, in [1] (Table 4), the power if the EU Council of Ministers from 1958 to 2007 is analyzed. We do that in two steps. First, uncertainty profiles [5] bounds in a macroscopic way the spread of the global uncertainty. Second, the associated $\mathfrak{a} / \mathfrak{d}$-games provides a balance of the uncertainty in between the best and the worst scenarios. Given an uncertainty profile $\mathcal{U}$ shaped to deal with Act, the $\mathfrak{a} / \mathfrak{d}$ game associated verifies the following: all the Nash equilibria have the same $\mathfrak{a}$ utility. Therefore we propose to take this utility as an assessment of the Coleman's power to act under uncertainty $\operatorname{Act}(\mathcal{U})$.

The paper is structured as follows. Section 2 is devoted to the preliminaries in weighted voting games and Coleman's power to act. In Section ?? we develop a perturbation strength model for weighted voting games. In particular the basic properties of the small perturbations case, PlusMinus, are developed. Section 3 devoted to uncertainty profiles and $\mathfrak{a} / \mathfrak{d}$ games in general. In Section ?? we develop the uncertainty in for majority voting games. Finally Section 7 is devoted to conclusions and open problems.

\section{Preliminaries}

A simple game $\Gamma$ is given by a tuple $(N, \mathcal{W})$ where $N$ is a set of $n$ players and $\mathcal{W}$ is a monotonic family of subsets of $N$ [?]. In the context of simple games, the subsets of $N$ 
are called coalitions, $N$ is the grand coalition and $S \in \mathcal{W}$ is a winning coalition. Any subset of $N$ which is not a winning coalition is called a losing coalition. The Coleman's power of the collectivity to act [2], is defined as $\operatorname{Act}(\Gamma)=\# \mathcal{W} / 2^{n}$. This measure of collective power $\operatorname{Act}(\Gamma)$ can be seen as the probability of the yes outcome assuming that all coalitions are equally like.

A weighted voting game [8] is a simple game defined by a tuple $\Gamma=\left\langle q ; w_{1}, \ldots w_{n}\right\rangle$, where $q$ is the quota and $w_{i} \in \mathbb{N}^{+}$is the weight of player $i$, for all $1 \leq i \leq n$. The set of players is $N=[n]=\{1, \ldots, n\}$. Let $w(S)=\sum_{i \in S} w_{i}$ denote the weight of coalition $S$. The set of winning coalitions is $\mathcal{W}(\Gamma)=\{S \mid w(S) \geq q\}$. Therefore, the set of losing coalitions is $\mathcal{L}(\Gamma)=\{S \mid w(S)<q\}$. Let us consider the case where the weights of all the players are equal, $\Gamma=\langle q ; w, \ldots, w\rangle$ with $n>1$. We denote such a game as $\Gamma_{n}(q ; w)$. Observe that, for $S \subseteq\{1, \ldots, n\}$ it holds $w(S)=w \times \# S$ and $\mathcal{W}(\Gamma)=\{S \mid w \times \# S \geq q\}$. In the case of equal weights, to catch the straight majority of of the total weight it is needed to capture a weight strictly greater than $\frac{n w}{2}$. In such a case we cannot distinguish between the requirement of having the majority of the players from having the majority of the players. In fact any quota between $\left\lfloor\frac{n w}{2}\right\rfloor+1$ and $\left.w\left\lfloor\frac{n}{2}\right\rfloor+1\right)$. We will consider two basic families of weighted voting games. The equal weight majority on $n$ players in which all the players have the same weight $w$, denote as $\Gamma(n, q, w)$ and the equal weight majority on $n$ players game as $\Gamma(n, w)=\Gamma(n, w\lfloor n / 2\rfloor+1, w)$. Observe that $\mathcal{W}(\Gamma(n, w))=\mathcal{W}(\Gamma(n, 1))$ $\operatorname{Act}(\Gamma(n, w))=\operatorname{Act}(\Gamma(n, 1))$.

In order to develop some examples, we consider the weights of the different states at the Council of Ministers of the EU (now the Council of the EU) along the time [1]. In 1958 the founding members were Germany (DE), France (FR), Italy (IT), The Netherlands (NL), Belgium (BE), and Luxembourg (LU) listed in non-decreasing order of assigned weights. The Council of Ministers at 1958 is summarized as $\Gamma_{\text {TheSix }}=$ $\langle 12 ; 4,4,4,2,2,1$,$\rangle . The quota was defined by a qualified majority rule, QMR, of the$ $70.6 \% 4$ that is $q=12 \approx 17 *(70.6 / 100)=12.002$. Along the period 1958 to 2014 the number of states increases from the initial 6 to $9,10,12,15,25$ and 27 . The voting system changed to cover the new arrivals. This leas to 25 different voting systems were used described by the following weighted voting games taken from [1].

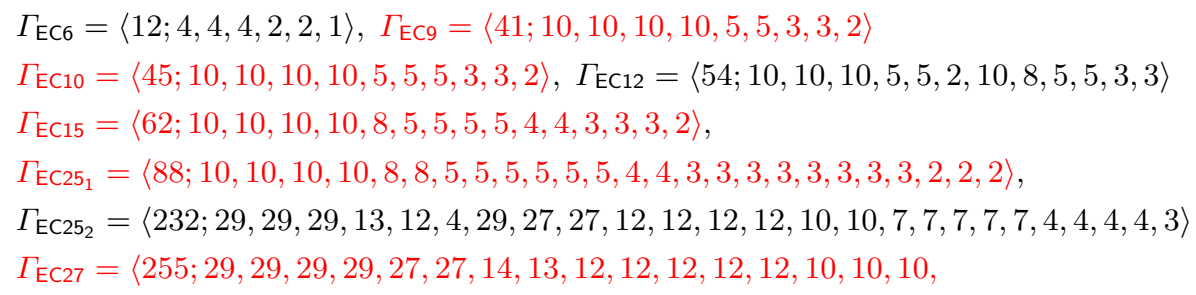

In all those games players 1 to 6 correspond to the six founding members in the same order as in the 1958 description. The parameters of those games and the Coleman's Power are: 


\begin{tabular}{|c|c|c|c|c|c|c|c|c|}
\hline & $\Gamma_{\mathrm{EC} 6}$ & $\Gamma_{\mathrm{EC} 9}$ & $\Gamma_{\mathrm{EC} 10}$ & $\Gamma_{\mathrm{EC} 12}$ & $\Gamma_{\mathrm{EC} 15}$ & $\Gamma_{\mathrm{EC} 25_{1}}$ & $\Gamma_{\mathrm{EU} 25_{2}}$ & $\Gamma_{\mathrm{EC} 27}$ \\
\hline Total $=w(N)$ & 17 & 58 & 63 & 76 & 87 & 124 & 321 & 345 \\
$\%$ & 70.6 & 70.7 & 71.4 & 71.1 & 71.3 & 71 & 72.3 & 73.9 \\
\hline$\# \mathcal{W}$ & 14 & 75 & 140 & 402 & 2549 & 1170000 & 1204448 & 2718774 \\
Power to Act & 0.2187 & 0.1464 & 0.1455 & 0.0981 & 0.0777 & 0.0348 & 0.0358 & 0.0202 \\
\hline
\end{tabular}

Note that the power to act is quite small and roughly decreases along the time.

\section{Uncertainty profiles and $\mathfrak{a} / \mathfrak{d}$ games}

Let us move on to adapt the definition of uncertainty profiles [5], [4]. They are useful to describe situations in which we have an approximate idea of the extension of the perturbation but we are uncertain over the specific location where it will impact the system. They are essentially based on three components: the set of weights that may be perturbed; the extent to which the perturbation can be applied; and the number of the components that can suffer perturbations. In an angel daemon framework we have two types of perturbations that will be exerted by $\mathfrak{a}$ towards increasing Act and by $\mathfrak{d}$ to decrease it.

In an $\mathfrak{a} / \mathfrak{d}$ framework two agents are considered: the angel (a), dealing with the optimistic side; and the daemon ( $\mathfrak{d})$, dealing with the pessimistic side of a perturbation. In our game, these agents act over the model by applying perturbations on the players' weights: the angels act in such a way to maximize the power to act and the daemon acts in order to minimize it. The perturbation values are real numbers, so they can be either positive or negative and we assume that both the angel and the daemon have limits in the influence they can exert.

Definition 1. A uncertain profile is a tuple $\mathcal{U}=\left\langle\Gamma, \mathcal{A}, \mathcal{D}, \delta_{\mathfrak{a}}, \delta_{\mathfrak{d}}, b_{\mathfrak{a}}, b_{\mathfrak{d}},\right\rangle$ where $\Gamma=$ $\left\langle q ; w_{1}, \ldots, w_{n}\right\rangle$ is a weighted voting game; $\mathcal{A}, \mathcal{D} \subseteq[n] ; \delta_{\mathfrak{a}}: \mathcal{A} \rightarrow \mathbb{Z}$ and $\delta_{\mathfrak{d}}: \mathcal{D} \rightarrow \mathbb{Z}$ represent the strength of the perturbation; $b_{\mathfrak{a}}, b_{\mathfrak{d}} \in \mathbb{N}$ are such that $b_{\mathfrak{a}} \leq \# \mathcal{A}$ and $b_{\mathfrak{d}} \leq \# \mathcal{D}$ and they represent the spread of the perturbation.

The exerted perturbation follows from joint actions $(a, d)$, verifying $a \subseteq \mathcal{A}, d \subseteq \mathcal{D}$ with $\# a=b_{\mathfrak{a}}$ and $\# d=b_{\mathfrak{d}}$. The effects of a joint action is a perturbed game $\Gamma[a, d]=$ $\left\langle q ; w_{1}^{\prime}, \ldots, w_{n}^{\prime}\right\rangle$ where

$$
w_{i}^{\prime}=w_{i}+ \begin{cases}0 & \text { if } i \notin a \cup d, \\ \delta_{\mathfrak{a}}(i) & \text { if } i \in a \backslash d, \\ \delta_{\mathfrak{d}}(i) & \text { if } i \in d \backslash a, \\ \delta_{\mathfrak{a}}(i)+\delta_{\mathfrak{d}}(i) & \text { if } i \in a \cap d\end{cases}
$$

To ensure $w_{i}^{\prime} \in \mathbb{N}^{+}$we require that $\left|\delta_{\mathfrak{a}}(i)\right|,\left|\delta_{\mathfrak{d}}(i)\right|<w_{i}$.

We are interested to know how $\mathfrak{a}$ and $\mathfrak{d}$ joint actions affect the number of winning coalitions through the perturbed game.

Example 1. Take $\mathcal{U}=\langle\langle 30 ; 10,10,10,5,5,2\rangle,\{1,5\},\{1,5\},(19,7),(-6,-3), 1,1\rangle$. Each joint action defines a perturbed game: $\Gamma[\{1\},\{1\}]=\langle 30 ; 23,10,10,5,5,2\rangle$ and $\Gamma[\{5\},\{5\}]=\langle 30 ; 10,10,10,5,9,2\rangle$ as an example. Observe that $\# \mathcal{W}(\Gamma[\{1\},\{1\}])=$ and $\# \mathcal{W}(\Gamma[\{5\},\{5\}])=17$. 
To asses the uncertainty of the power to act in an uncertainty profile we introduce an strategic zero-sum $\mathfrak{a} / \mathfrak{d}$ game and use the value of this associated game.

Definition 2. Given $\mathcal{U}=\left\langle\Gamma, \mathcal{A}, \mathcal{D}, \delta_{\mathfrak{a}}, \delta_{\mathfrak{d}}, b_{\mathfrak{a}}, b_{\mathfrak{d}},\right\rangle$, the associated angel/daemon (or $\mathfrak{a} / \mathfrak{d})$ game is $\Gamma(\mathcal{U})=\left\langle\{\mathfrak{a}, \mathfrak{d}\}, A_{\mathfrak{a}}, A_{\mathfrak{d}}, u_{\mathfrak{a}}, u_{\mathfrak{d}}\right\rangle$. Game $\Gamma(\mathcal{U})$ has two players: the angel $\mathfrak{a}$ and the daemon $\mathfrak{d}$. The player's actions are $A_{\mathfrak{a}}=\left\{a \subseteq \mathcal{A} \mid \# a=b_{\mathfrak{a}}\right\}$ and $A_{\mathfrak{d}}=$ $\left\{d \subseteq \mathcal{D} \mid \# d=b_{\mathfrak{d}}\right\}$. For $(a, d) \in A_{\mathfrak{a}} \times A_{\mathfrak{d}}$ utilities are $u_{\mathfrak{a}}(a, d)=\# \mathcal{W}(\Gamma[a, d])$ and $u_{\mathfrak{d}}(a, d)=-u_{\mathfrak{a}}(a, d)$.

Notice that, in an $\mathfrak{a} / \mathfrak{d}$ game the set of strategy profiles is $A_{\mathfrak{a}} \times A_{\mathfrak{d}}$. A player's choice of actions can be done probabilistically. Mixed strategies for $\mathfrak{a}$ and $\mathfrak{d}$ are probability distributions $\alpha: A_{\mathfrak{a}} \rightarrow[0,1]$ and $\beta: A_{\mathfrak{d}} \rightarrow[0,1]$ respectively. A mixed strategy is a tuple $(\alpha, \beta)$ such that $u_{\mathfrak{p}}(\alpha, \beta)=\sum_{(a, d) \in A_{\mathfrak{a}} \times A_{\mathfrak{d}}} \alpha(a) u_{\mathfrak{p}}(a, d) \beta(d)$ for $\mathfrak{p} \in\{\mathfrak{a}, \mathfrak{d}\}$. Let $\Delta_{\mathfrak{a}}$ and $\Delta_{\mathfrak{d}}$ denote the set of mixed strategies for players $\mathfrak{a}$ and $\mathfrak{d}$, respectively. A pure strategy profile $(a, d)$ is a special case of mixed strategy profile $(\alpha, \beta)$ in which $\alpha(a)=1$ and $\beta(d)=1$. A mixed strategy profile $(\alpha, \beta)$ is a Nash equilibrium if for any $\alpha^{\prime} \in \Delta_{\mathfrak{a}}$ it holds $u_{\mathfrak{a}}(\alpha, \beta) \geq u_{\mathfrak{a}}\left(\alpha^{\prime}, \beta\right)$ and for any $\beta^{\prime} \in \Delta_{\mathfrak{d}}$ it holds $u_{\mathfrak{d}}(\alpha, \beta) \geq$ $u_{\mathfrak{d}}\left(\alpha, \beta^{\prime}\right)$. A pure Nash equilibrium, PNE, is a Nash equilibrium $(a, d)$ where $a$ and $d$ are pure strategies.

Example 2. SHOULD BE A FOLLOW-UP OF current Ex 1 Given $\mathcal{U}$ in Example 4 actions are $A_{\mathfrak{a}}=A_{\mathfrak{d}}=\{\{1\},\{2\}\}$ and $\Gamma(\mathcal{U})$ is given by the utility of the row player, $\mathfrak{a}$, in the following table

$\{1\}$

with PNE $=\{(\{2\},\{2\})\}$

It is well known that all Nash equilibria of zero-sum game $G$ have the same value $\nu(G)$ corresponding to the utility of the row player. Considering that in $\mathfrak{a} / \mathfrak{d}$ games the row player id $\mathfrak{a}$ we can extend the Coleman's power to act to an uncertainty profile $\mathcal{U}$ as follows.

Definition 3. Let $\mathcal{U}=\left\langle\Gamma, \mathcal{A}, \mathcal{D}, \delta_{\mathfrak{a}}, \delta_{\mathfrak{d}}, b_{\mathfrak{a}}, b_{\mathfrak{d}}\right\rangle$, be an uncertainty profile, the Coleman's power to act of $\mathcal{U}$ is $\operatorname{Act}(\mathcal{U})=\# \mathcal{W}(\mathcal{U}) / 2^{n}$.

Observe that according to $\operatorname{Act}(\mathcal{U})$ is the Coleman's power to act of a weighted voting game when $G(\mathcal{U})$ has a PNE or an average of the Coleman's power to act of several games, under a NE distribution. When $(\alpha, \beta)$ is a Nash equilibrium of $G(\mathcal{U})$ the $\mathfrak{a}$ utility is $\sum_{(a, d) \in A_{a} \times A_{\mathfrak{⿰}}} \alpha(a)\left(\# \mathcal{W}\left(\Gamma_{\mathcal{S}}[a, d]\right)\right) \beta(d)$ and therefore we get the expression $\operatorname{Act}(\mathcal{U})=\sum_{(a, d) \in A_{\mathfrak{a}} \times A_{\mathfrak{o}}} \alpha(a)\left(\operatorname{Act}\left(\Gamma_{\mathcal{S}}[a, d]\right)\right) \beta(d)$.

Example 3. SHOULD BE A FOLLOW-UP OF previous or some with a non pure NE

Example 4. MAKES ANY SENSE NOW? Take $\mathcal{U}=\left\langle\langle 30 ; 5,10,10,10,2,5\rangle, \mathcal{S}_{\text {Six }}, \mathcal{A}, \mathcal{D}, 1,1, u_{\mathfrak{a}}, u_{\mathfrak{d}}\right\rangle$ based on Example 8 where $\mathcal{A}=\mathcal{D}=\{1,2\}$ and $u_{\mathfrak{a}}(a, d)=\# \mathcal{W}\left(\Gamma_{\mathcal{S}_{\text {Six }}}[a, d]\right)$, $u_{\mathfrak{0}}(a, d)=\# \mathcal{L}\left(\Gamma_{\mathcal{S}_{\text {Six }}}(a, d]\right)$. For instance $u_{\mathfrak{a}}(\{1\},\{1\})=\# \mathcal{W}(\langle 30 ; 9,10,10,10,2,5\rangle)=$ 17 . 


\section{Majority games with equal weights}

We consider first the simplest case of weighted games in which all the players have equal weight, $\Gamma(n, q, w)$ or $\Gamma(n, w)$. Let us consider weighted voting games that can be seen as a small perturbations of $\Gamma_{n}(n, w)$. We start just perturbing the weight of the two players with opposite strengths. By straightforward combinatorial arguments we get:

Lemma 1. check that the statement is correct changed $\delta=1$ to $0<\delta<w$. Let $n>2, w>1$ and $0<\delta<w, i, j \in[n]$ so that $i \neq j$. Let $\Gamma=\Gamma(n, w)$ and $\mathcal{U}(\delta ; i, j)=\langle\Gamma,\{i\},\{j\},(\delta),(-\delta), 1,1\rangle$ and $\Gamma^{\prime}=\Gamma[\{i\},\{j\}]$. Then, we have

$$
\mathcal{W}\left(\Gamma^{\prime}\right)=\{S \mid \# S \geq\lfloor n / 2\rfloor+1\} \cup\{\{2\} \cup S \mid S \subseteq N \backslash\{i, j\}, \# S=\lfloor n / 2\rfloor-1\}
$$

and the number of winning coalitions is

$$
\# \mathcal{W}\left(\Gamma^{\prime}\right)=\# \mathcal{W}(\Gamma(n, w))+\left(\begin{array}{c}
n-2 \\
\lfloor n / 2\rfloor-1
\end{array}\right)
$$

Example 5. Take $n=3, w=2$ and $\delta=1$. In such a case $w\lfloor n / 2\rfloor+1=3$ and $\left.\Gamma_{(} 3,2\right)=\langle 3 ; 2,2,2\rangle$. Under $\mathcal{U}(\delta ; i, j)$ we have $\Gamma[\{1\},\{2\}]=\langle 3 ; 1,3,2\rangle$. The sets of winning coalitions are $\mathcal{W}(\Gamma(3,2)=\{\{1,2\},\{1,3\},\{2,3\},\{1,2,3\}\}$ and

$\mathcal{W}(\Gamma[\{1\},\{2\}]=\mathcal{W}(\Gamma(3,2)) \cup\{\{2\}\}$.

In order to provide intuition on the use of $\mathfrak{a}$ and $\mathfrak{d}$ we analyze now the case with equal and opposite strengths. Let $n>2, w>1,0<|\delta|<w \Gamma$, and let $(\mathcal{A}, \mathcal{D} \subseteq$ $[n]$, the minimal egalitarian uncertainty profile is defined as $\mathcal{M E}(n, w, \delta ; \mathcal{A}, \mathcal{D})=$ $\left\langle\Gamma(n, w), \mathcal{A}, \mathcal{D}, \delta_{\mathfrak{a}}, \delta_{\mathfrak{d}}, 1,1\right\rangle$, where $\delta_{\mathfrak{a}}(i)=\delta$, for $i \in \mathcal{A}$, and $\delta_{\mathfrak{d}}(i)=-\delta$, for $i \in \mathcal{D}$.

Lemma 2. Let $n>2, w>1$ and $0<\delta<w$, let $\mathcal{A}, \mathcal{D} \subseteq[n]$. Let $\Gamma=\Gamma(n, w)$ and $\mathcal{U}=\mathcal{M E}(n, w, \delta ; \mathcal{A}, \mathcal{D})$, then we have $\# \mathcal{W}(\Gamma[\{i\},\{i\}])=\# \mathcal{W}(\Gamma(w, n))$, for $i \in \mathcal{A} \cap \mathcal{D}$ and $\# \mathcal{W}(\Gamma[\{i\},\{j\}]=\# \mathcal{W}(\Gamma[\{k\},\{\ell\}] \geq \# \mathcal{W}(\Gamma(w, n))$, for $i, k \in \mathcal{A}$, $j . \ell \in \mathcal{D}, i \neq j$ and $k \neq \ell$.

Proof. Observe that when both $\mathfrak{a}$ and $\mathfrak{d}$ select a common player $\{i\}$ the two perturbations cancel and the so obtained game id the initial one. When $(a, d)=(\{i\},\{j\})$ with $i \neq j$ the perturbed game has one player with weight $w+\delta$, another with weight $w-\delta$ and the remaining players with weight $w$. Thus the game is the same up to a permutation of the players and in such a case the number of winning coallitions is the same. The last inequality follows from Lemma 1.

Theorem 1. Let $n>2$ and $w>1$ and $0<\delta<w$, let $\mathcal{A}, \mathcal{D} \subseteq[n]$. Assume that $\# \mathcal{A}>0, \# \mathcal{D}>0$ Let $\Gamma=\Gamma(n, w)$ and $\mathcal{U}=\mathcal{M E}(n, w, \delta ; \mathcal{A}, \mathcal{D})$. Then, if $\mathcal{A}=\mathcal{D}$, $\Gamma(\mathcal{U})$ has no PNE. If $\mathcal{A} \neq \mathcal{D}$ and $\mathcal{A} \subseteq \mathcal{D} \operatorname{PNE}(\Gamma(\mathcal{U}))=\{(\{i\},\{i\}) \mid i \in \mathcal{A}, j \in \mathcal{D}\}$ and $\operatorname{Act}(\mathcal{U})=\operatorname{Act}(\Gamma(n, w)$, otherwise $\operatorname{PNE}(\Gamma(\mathcal{U}))=\{(\{i\},\{i\}) \mid i \in \mathcal{A} \backslash \mathcal{D}, j \in \mathcal{D}\}$ and $\operatorname{Act}(\mathcal{U})=\operatorname{Act}\left(\Gamma(n, w)+\frac{1}{2^{n}}\left(\begin{array}{c}n-2 \\ \lfloor n / 2\rfloor-1\end{array}\right)\right.$. 
Proof. From Def. 2, $A_{\mathfrak{a}} \times A_{\mathfrak{d}}=\{(\{i\},\{j\}) \mid(i, j) \in \mathcal{A} \times \mathcal{D}\}$ as by Lemma 2 all perturbed games are only of two types $\Gamma[\{i\},\{i\}]$ or $\Gamma[\{i\},\{j\}], j \neq i$. If $\mathcal{A} \subseteq \mathcal{D}$, for each action $\{i\}$ of $\mathfrak{a}, \mathfrak{d}$ best response is $\{i\}$. Otherwise, for each action $\{j\}$ of $\mathfrak{d}, \mathfrak{a}$ best response is $\{i\}$ for some $i \notin \mathcal{D}$. This provides the characterization of the PNE. The later expression follows from Lemma 1 .

Let us conclude with an example in which the $\mathfrak{a} / \mathfrak{d}$ game has no PNE but exactly one NE.

Example 6. Let $n>2$ and $w>1$. let $\Gamma=\Gamma(n, w)$ and $\mathcal{U}=\mathcal{M E}(n, w, 1 ;\{1,2\},\{1,2\})$, by Theorem 1 we know that $G(\mathcal{U})$ has no PNE. As $b_{\mathfrak{a}}=b_{\mathfrak{d}}=1$ we have $A_{\mathfrak{a}}=A_{\mathfrak{d}}=$ $\{\{1\},\{2\}\}$ and $\mathfrak{a}$ 's payoff matrix in the $\mathfrak{a} / \mathfrak{d}$ game is

$\{1\}$

\begin{tabular}{|c|c|}
\hline$\# \mathcal{W}(\Gamma(n, w))$ & $\# \mathcal{W}\left(\Gamma(n, w)+\frac{1}{2^{n}}\left(\begin{array}{c}n-2 \\
\lfloor n / 2\rfloor-1\end{array}\right)\right.$ \\
\hline$\# \mathcal{W}\left(\Gamma(n, w)+\frac{1}{2^{n}}\left(\begin{array}{c}n-2 \\
\lfloor n / 2\rfloor-1\end{array}\right)\right.$ & $\# \mathcal{W}(\Gamma(n, w))$ \\
\hline
\end{tabular}

It is easy to see that the unique (mixed) Nash equilibrium is $(\alpha, \beta)=((1 / 2,1 / 2),(1 / 2,1 / 2))$. Therefore, $\operatorname{Act}(\mathcal{U})=\operatorname{Act}(\Gamma(n, w))+\frac{1}{2^{n-1}}\left(\begin{array}{c}n-2 \\ \lfloor n / 2\rfloor-1\end{array}\right)$.

\section{Computational complexity considerations}

Observe that the number of wining coalitions in a weighted voting games corresponds to counting the number of solutions of a Knapsack problem which is known to be a \#Pcomplete problem []. We analyze here the extent to which such hardness result can be extended to computing the value of the associated $\mathfrak{a} / \mathfrak{d}$ games and related computational problems. In particular we consider the following problems:

- EQACT: Given two weighted voting games $\Gamma$ and $\Gamma^{\prime}$ on $n$ players, is $\operatorname{Act}(\Gamma)=$ $\operatorname{Act}\left(\Gamma^{\prime}\right)$.

- IsBestR Given an uncertainty profile $\mathcal{U}$ and a joint action $(a, d) \in A_{\mathfrak{a}} \times \mathfrak{o}$, is $d$ a best response to $a$ in $G(\mathcal{U})$ ?

- VALUE Given an uncertainty profile $\mathcal{U}$, compute $\nu(G(\mathcal{U}))$.

Theorem 2. EQACT is co-NP-hard and can be solved in pseudopolynomial time. IsBESTR is NP-hard. try to complete this!

\section{A study based on the Council of Ministers of the EC}

Observe that computing the number of wining coalitions can be done in pseudopolynomial time using Dynamic Programming on an array of size $\sum_{i=1}^{n} w_{i}$. However even 
when the weights are polynomial in the number of players the number of strategies can still be exponential. However, when the weights (initial and perturbed) are polynomial in $n$ and the spread is constant it is possible to compute a complete description of $G(\mathcal{U})$ and using a LP solver obtaining the value of the game. This has been our approach to develop de following examples. Our objective is to analyze the assessments of different uncertainty profiles. We also want to explore the differences in the assessment when in the perturbed weighted voting game instead of preserving the quota we keep only its proportionality to the total weight. For doing so we asses different uncertainty profile for the 25 voting systems of the EC when the uncertainty fells on top of the weights of the six founder members.

Example 7. Consider the weights of the funding states in EC6, EC12 and EC27. We can see the weights of EC6 and EC27 as obtained by perturbations of those of EC12. $\mathfrak{a}$ moves forward the weights to those in EC27 while $\mathfrak{d}$ wishes to move them back to EC6. Assuming the $\mathcal{A}=\mathcal{B}=\{1, \ldots, 6\}$, we get the expressions:

\begin{tabular}{|c||c|c|c|c|c|c|}
\hline & DE & FR & IT & NL & LU & BE \\
\hline EC6 & 4 & 4 & 4 & 2 & 2 & 1 \\
EC12 & 10 & 10 & 10 & 5 & 5 & 2 \\
EC27 & 29 & 29 & 29 & 13 & 12 & 4 \\
\hline$\delta_{12-27}$ & 19 & 19 & 19 & 8 & 7 & 2 \\
$\delta_{12-6}$ & -6 & -6 & -6 & -3 & -3 & -1 \\
\hline
\end{tabular}

We assesed uncertainty profiles of the form $\left.\mathcal{U}_{12}(b)=\left\langle\Gamma_{\mathrm{EC} 12},[6],[6], \delta_{12-27}, \delta_{12-6}, b, b\right\rangle\right\rangle$ our results are summarized in the following table.

TBD

Example 8. From previous draft Focusing on the initial six in EC12 and keeping a $\%$ of 71.1 we get a quota of 30 . The restriction of the six inside EC12 is described by $\Gamma=\langle 30 ; 5,10,10,10,2,5\rangle$. Given $\mathcal{S}_{\mathrm{Six}}$ in Example 7 we consider some cases of $\Gamma_{\mathcal{S}_{\text {six }}}(a, d)$. To do that we need to pay attention to $\delta_{\mathcal{S}_{\text {six }}}(i)[a, d]$. For instance, take $(a, d)=(\{1\},\{1\})$ where 1 corresponds to Belgium. As $i \in a \cap d$, according to Definition ?? we have $\delta_{\mathcal{S}_{\text {Six }}}(1)[\{1\},\{1\}]=\delta_{\mathfrak{a}}(1)+\delta_{\mathfrak{d}}(1)=7-3=4$ and $w_{1}[\{1\},\{1\}]=$ $w_{1}+4=9$. For $i \in\{2,6\}$ we have $i \notin a \cup d$ and therefore $w_{i}^{\prime}=w_{i}$. Therefore $\Gamma_{\mathcal{S}_{\text {Six }}}(\{1\},\{1\})=\langle 30 ; 9,10,10,10,2,5\rangle$. Consider $(a, d)=(\{1\},\{2\})$ where 2 corresponds to France. As $1 \in a \backslash d$ it holds $w_{1}^{\prime}=w_{1}+\delta_{\mathfrak{a}}(1)=12$. As $2 \in d \backslash a$ it holds $w_{2}^{\prime}=w_{2}+\delta_{\mathfrak{d}}(2)=4$. As other weights remain unchanged, $\Gamma_{\mathcal{S}_{\text {Six }}}(\{1\},\{2\})=$ $\langle 30 ; 12,4,10,10,2,5\rangle$.

\section{Conclusions and open problems}

In order to tackle with the uncertainty in Coleman's power to act Act issued from the imprecisions on weights in voting games we introduce uncertainty profiles and define $\operatorname{Act}(\mathcal{U})$. We have proved the uniqueness of $\operatorname{Act}(\mathcal{U})$ and developed several properties and examples. 
Other topics merits to be studied. in particular in [2] two other measures are also considered for any player $i$ of a weighted voting game $\Gamma$. The power to initiate action, Initiate $_{i}(\Gamma)=\#\{S \in \mathcal{L}(\Gamma) \mid S \cup\{i\} \in \mathcal{W}(\Gamma)\} / \# \mathcal{L}(\Gamma)$. The power of $i$ to initiate action give us the likelihood that $i$ turns a loosing coalition into a winning one. The power to prevent action, $\left.\operatorname{Prevent}_{i}(\Gamma)=\#\{S \in \mathcal{W}(\Gamma) \mid S \backslash\{i\} \in \mathcal{L}(\Gamma)\}\right) / \# \mathcal{W}(\Gamma)$. The power of $i$ to prevent action is the fraction of winning coalitions for which $i$ is critical. It remains open how to deal with with the power to initiate or the power to prevent in an uncertain environment. This will allow us to compare with the probabilistic approach undertaken in [1]. It will be also interested to get uncertainty profiles well adapted to the Shapley values. This will allow us to compare with probabilistic approaches like the one given in [3]. This will allow us to compare with much more practical approaches like those given in [6] and [1].

\section{References}

1. N. Antonakakis, H. Badinger, and W. H. Reuter. From Rome to Lisbon and beyond: Member States' power, efficiency, and proportionality in the EU Council of Ministers. Working Paper 175, Vienna University of Economics and Business, Department of Economics, 2014.

2. J. Coleman. In B. Lieberman, editor, Social choice, chapter Control of collectivities and the power of a collectivity to act, pages 269-300. Gordon and Breach, 1971. Reedited in Routledge Revivals, 2011.

3. S. Shaheen Fatima, M. Wooldridge, and N. R. Jennings. An analysis of the shapley value and its uncertainty for the voting game. In M. P. Gleizes, G. A. Kaminka, A. Nowé, S. Ossowski, K. Tuyls, and K. Verbeeck, editors, EUMAS 2005, Belgium, December 7-8, 2005, pages 480481. Koninklijke Vlaamse Academie van Belie voor Wetenschappen en Kunsten, 2005.

4. J. Gabarro and M. Serna. Uncertainty in basic short-term macroeconomic models with angeldaemon games. Int. J. of Data Analysis Techniques and Strategies. To appear.

5. J. Gabarro, M. Serna, and A. Stewart. Analysing web-orchestrations under stress using uncertainty profiles. Comput. J., 57(11):1591-1615, 2014.

6. Elena Mielcova. The uncertainty in voting power: The case of the czech parliament 19962004. AUCO Czech Economic Review, 4(2):201-221, 2010.

7. Lloyd Shapley. In H.W. Kuhn and A.W. Tucker, editors, Contributions to the Theory of Games, volume II, chapter A Value for n-person Games, pages 307-317. Princeton University Press, 1953. Included in Classics in Game Theory.

8. Lloyd Shapley. Simple games: An outline of the descriptive theory. Systems Research and Behavioral Science, 7(1):59-66, 1962. 